TRANSACTIONS OF THE

AMERICAN MATHEMATICAL SOCIETY

Volume 361, Number 4, April 2009, Pages 1853-1865

S 0002-9947(08)04707-7

Article electronically published on October 20, 2008

\title{
ALGEBRAIC SHIFTING AND GRADED BETTI NUMBERS
}

\author{
SATOSHI MURAI AND TAKAYUKI HIBI
}

\begin{abstract}
Let $S=K\left[x_{1}, \ldots, x_{n}\right]$ denote the polynomial ring in $n$ variables over a field $K$ with each $\operatorname{deg} x_{i}=1$. Let $\Delta$ be a simplicial complex on $[n]=$ $\{1, \ldots, n\}$ and $I_{\Delta} \subset S$ its Stanley-Reisner ideal. We write $\Delta^{e}$ for the exterior algebraic shifted complex of $\Delta$ and $\Delta^{c}$ for a combinatorial shifted complex of $\Delta$. Let $\beta_{i i+j}\left(I_{\Delta}\right)=\operatorname{dim}_{K} \operatorname{Tor}_{i}\left(K, I_{\Delta}\right)_{i+j}$ denote the graded Betti numbers of $I_{\Delta}$. In the present paper it will be proved that (i) $\beta_{i i+j}\left(I_{\Delta^{e}}\right) \leq \beta_{i i+j}\left(I_{\Delta^{c}}\right)$ for all $i$ and $j$, where the base field is infinite, and (ii) $\beta_{i i+j}\left(I_{\Delta}\right) \leq \beta_{i i+j}\left(I_{\Delta^{c}}\right)$ for all $i$ and $j$, where the base field is arbitrary. Thus in particular one has $\beta_{i i+j}\left(I_{\Delta}\right) \leq \beta_{i i+j}\left(I_{\Delta^{l e x}}\right)$ for all $i$ and $j$, where $\Delta^{\text {lex }}$ is the unique lexsegment simplicial complex with the same $f$-vector as $\Delta$ and where the base field is arbitrary.
\end{abstract}

\section{INTRODUCTION}

Kalai [8] together with Herzog [7] offer an attractive introduction, which includes several unsolved problems and conjectures, to the combinatorial and algebraic study of shifting theory in algebraic and extremal combinatorics.

Let $S=K\left[x_{1}, \ldots, x_{n}\right]$ denote the polynomial ring in $n$ variables over a field $K$ with each $\operatorname{deg} x_{i}=1$. One of the current trends in computational commutative algebra is the computation of the graded Betti numbers of homogeneous ideals. Recall that the graded Betti numbers $\beta_{i j}=\beta_{i j}(I)$, where $i, j \geq 0$, of a homogeneous ideal $I \subset S$ are

$$
\beta_{i j}(I)=\operatorname{dim}_{K} \operatorname{Tor}_{i}(K, I)_{j} .
$$

In other words, the graded Betti numbers $\left\{\beta_{i j}\right\}_{i, j=0,1, \ldots}$ appear in the minimal graded free resolution

$$
0 \longrightarrow \bigoplus_{j} S(-j)^{\beta_{h j}} \longrightarrow \cdots \longrightarrow \bigoplus_{j} S(-j)^{\beta_{1 j}} \longrightarrow \bigoplus_{j} S(-j)^{\beta_{0 j}} \longrightarrow I \longrightarrow 0
$$

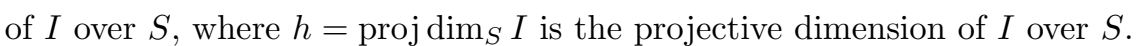

Let $\Delta$ be a simplicial complex on $[n]=\{1, \ldots, n\}$ and $I_{\Delta} \subset S$ the StanleyReisner ideal of $\Delta$. We write $\Delta^{s}, \Delta^{e}$ and $\Delta^{c}$ for the symmetric algebraic shifted complex, the exterior algebraic shifted complex and a combinatorial shifted complex, respectively, of $\Delta$. Since the paper [1] was published, it has been conjectured that for an arbitrary simplicial complex $\Delta$ on $[n]$ one has

$$
\beta_{i i+j}\left(I_{\Delta}\right) \leq \beta_{i i+j}\left(I_{\Delta^{s}}\right) \leq \beta_{i i+j}\left(I_{\Delta^{e}}\right) \leq \beta_{i i+j}\left(I_{\Delta^{c}}\right)
$$

Received by the editors March 2, 2007.

2000 Mathematics Subject Classification. Primary 13D02; Secondary 13F55.

(C)2008 American Mathematical Society 
for all $i$ and $j$. When the base field is of characteristic 0 , the first inequality $\beta_{i i+j}\left(I_{\Delta}\right) \leq \beta_{i i+j}\left(I_{\Delta s}\right)$ is proved in [3, Theorem 2.1].

Let $\Delta^{\prime}$ be a shifted (or strongly stable [1, p. 365]) simplicial complex with the same $f$-vector as $\Delta$ and $\Delta^{\text {lex }}$ the unique lexsegment simplicial complex with the same $f$-vector as $\Delta$ ([1, Theorem 3.5]). It is known [1, Theorem 4.4] that $\beta_{i i+j}\left(I_{\Delta^{\prime}}\right) \leq \beta_{i i+j}\left(I_{\Delta^{\text {lex }}}\right)$. Since $\Delta^{s}$ is shifted with the same $f$-vector as $\Delta$, when the base field is of characteristic 0 , one has $\beta_{i i+j}\left(I_{\Delta}\right) \leq \beta_{i i+j}\left(I_{\Delta^{\operatorname{lex}}}\right)$ for all $i$ and $j$ ([3, Theorem 2.9]).

The main purpose of the present paper is to establish two fundamental results stated below concerning the graded Betti numbers of $I_{\Delta}, I_{\Delta^{e}}$ and $I_{\Delta^{c}}$.

Theorem 2.10. Let the base field be infinite. Let $\Delta$ be a simplicial complex, $\Delta^{e}$ the exterior algebraic shifted complex of $\Delta$ and $\Delta^{c}$ a combinatorial shifted complex of $\Delta$. Then

$$
\beta_{i i+j}\left(I_{\Delta^{e}}\right) \leq \beta_{i i+j}\left(I_{\Delta^{c}}\right)
$$

for all $i$ and $j$.

Theorem 3.4. Let the base field be arbitrary. Let $\Delta$ be a simplicial complex and $\Delta^{c}$ a combinatorial shifted complex of $\Delta$. Then

$$
\beta_{i i+j}\left(I_{\Delta}\right) \leq \beta_{i i+j}\left(I_{\Delta^{c}}\right)
$$

for all $i$ and $j$.

Since $\Delta^{c}$ is shifted with the same $f$-vector as $\Delta$, it follows from Theorem 3.4 together with [1, Theorem 4.4] that

Corollary 3.5. Let the base field be arbitrary. Let $\Delta$ be a simplicial complex and $\Delta^{\text {lex }}$ the unique lexsegment simplicial complex with the same $f$-vector as $\Delta$. Then

$$
\beta_{i i+j}\left(I_{\Delta}\right) \leq \beta_{i i+j}\left(I_{\Delta^{\operatorname{lex}}}\right)
$$

for all $i$ and $j$.

The present paper will be organized as follows. First of all, following [7 the fundamental materials on algebraic shifting will be summarized in Section 1. Second, our proof of Theorem 2.10 will be achieved in Section 2. On the other hand, based on Hochster's formula [4, Theorem 5.5.1] to compute graded Betti numbers of Stanley-Reisner ideals, we will prove Theorem 3.4 in Section 3.

Finally, in Section 4 the bad behavior of graded Betti numbers of $I_{\Delta^{c}}$ will be studied. More precisely, since a combinatorial shifted complex of $\Delta$ is not unique, it is natural to ask, given a simplicial complex $\Delta$, if there exist combinatorial shifted complexes $\Delta_{b}^{c}$ and $\Delta_{\sharp}^{c}$ of $\Delta$ such that, for each combinatorial shifted complex $\Delta^{c}$ of $\Delta$ and for all $i$ and $j$, one has

$$
\beta_{i i+j}\left(I_{\Delta_{b}^{c}}\right) \leq \beta_{i i+j}\left(I_{\Delta^{c}}\right) \leq \beta_{i i+j}\left(I_{\Delta_{\sharp}^{c}}\right) .
$$

Unfortunately, in general, the existence of such combinatorial shifted complexes $\Delta_{b}^{c}$ and $\Delta_{\sharp}^{c}$ cannot be expected (Theorem 4.3). In particular, we construct a simplicial complex $\Delta$ for which there is no combinatorial shifted complex $\Delta^{c}$ of $\Delta$ with $\Delta^{e}=$ $\Delta^{c}$ (Corollary 4.4). 


\section{Algebraic shifting}

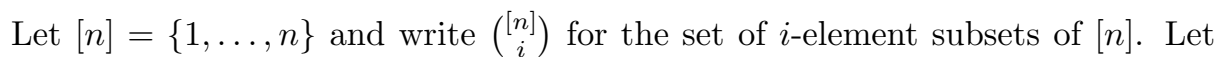
$S=K\left[x_{1}, \ldots, x_{n}\right]$ denote the polynomial ring in $n$ variables over a field $K$ with each $\operatorname{deg} x_{i}=1$. Let $V$ be a vector space over $K$ of dimension $n$ with basis $e_{1}, \ldots, e_{n}$ and $E=\bigoplus_{d=0}^{n} \wedge^{d}(V)$ the exterior algebra of $V$. If $\sigma=\left\{j_{1}, \ldots, j_{d}\right\} \in\left(\begin{array}{c}{[n]} \\ d\end{array}\right)$ with $j_{1}<\cdots<j_{d}$, then $x_{\sigma}=x_{j_{1}} \cdots x_{j_{d}}$ is a squarefree monomial of $S$ of degree $d$ and $e_{\sigma}=e_{j_{1}} \wedge \cdots \wedge e_{j_{d}} \in \wedge^{d}(V)$ will be called a monomial of $E$ of degree $d$.

Let $\Delta$ be a simplicial complex on $[n]$. Thus $\Delta$ is a collection of subsets of $[n]$ such that (i) $\{j\} \in \Delta$ for all $j \in[n]$ and (ii) if $\tau \subset[n]$ and $\sigma \in \Delta$ with $\tau \subset \sigma$, then $\tau \in \Delta$. A face of $\Delta$ is an element $\sigma \in \Delta$. The f-vector of $\Delta$ is the vector $f(\Delta)=\left(f_{0}, f_{1}, \ldots\right)$, where $f_{i}$ is the number of faces $\sigma \in \Delta$ with $|\sigma|=i+1$. (For a finite set $\sigma$ the notation $|\sigma|$ stands for its cardinality.) The Stanley-Reisner ideal of $\Delta$ is the ideal $I_{\Delta}$ of $S$ generated by those squarefree monomials $x_{\sigma}$ with $\sigma \notin \Delta$. The exterior face ideal of $\Delta$ is the ideal $J_{\Delta}$ of $E$ generated by those monomials $e_{\sigma}$ with $\sigma \notin \Delta$.

If $I \subset S$ is a squarefree ideal, i.e., an ideal generated by squarefree monomials, with each $x_{i} \notin I$, then there is a unique simplicial complex $\Delta$ on $[n]$ with $I=I_{\Delta}$. If $I \subset E$ is a monomial ideal, i.e., an ideal generated by monomials, with each $e_{i} \notin I$, then there is a unique simplicial complex $\Delta$ on $[n]$ with $I=J_{\Delta}$.

A monomial ideal $I \subset S$ is called strongly stable if for each monomial $u \in I$ and for each $j \in[n]$ for which $x_{j}$ divides $u$ one has $x_{i} u / x_{j} \in I$ for all $i<j$. A squarefree ideal $I \subset S$ is called squarefree strongly stable if for each monomial $x_{\sigma} \in I$ and for each $j \in \sigma$ one has $x_{(\sigma \backslash\{j\}) \cup\{i\}} \in I$ for all $i<j$ with $i \notin \sigma$. A monomial ideal $I \subset E$ is called strongly stable if for each monomial $e_{\sigma} \in I$ and for each $j \in \sigma$ one has $e_{(\sigma \backslash\{j\}) \cup\{i\}} \in I$ for all $i<j$ with $i \notin \sigma$.

We say that a simplicial complex $\Delta$ on $[n]$ is shifted if the monomial ideal $J_{\Delta}$ is strongly stable (or equivalently, the squarefree ideal $I_{\Delta}$ is squarefree strongly stable). In other words, $\Delta$ is shifted if $\Delta$ possesses the property that for each face $\sigma \in \Delta$ and for each $i \in \sigma$ one has $(\sigma \backslash\{i\}) \cup\{j\} \in \Delta$ for all $j>i$ with $j \notin \sigma$.

Assume that the base field $K$ is of characteristic 0 . Fix the reverse lexicographic order $<_{\text {rev }}$ on $S=K\left[x_{1}, \ldots, x_{n}\right]$ induced by the ordering $x_{1}>\cdots>x_{n}$. Given a homogeneous ideal $I \subset S$, we write $\operatorname{Gin}^{S}(I)$ for the generic initial ideal [6, p. 129] of $I$ with respect to $<_{\text {rev }}$. The generic initial ideal $\operatorname{Gin}^{S}(I)$ of a homogeneous ideal $I \subset S$ is strongly stable [6, Theorem 1.27].

We refer the reader to 2 for the foundation on the Gröbner basis theory in the exterior algebra. Assume that the base field $K$ is infinite. We work with the reverse lexicographic order $<_{\text {rev }}$ on $E$ induced by the ordering $e_{1}>e_{2}>\cdots>e_{n}$. Given a homogeneous ideal $I \subset E$, we write $\operatorname{Gin}^{E}(I)$ for the generic initial ideal [2, p. 183] of $I$ with respect to $<_{\text {rev }}$. The generic initial ideal $\operatorname{Gin}^{E}(I)$ of a homogeneous ideal $I \subset E$ is strongly stable [2, Proposition 1.7].

A shifting operation on $[n]$ is a map which associates each simplicial complex $\Delta$ on $[n]$ with a simplicial complex $\operatorname{Shift}(\Delta)$ on $[n]$ and which satisfies the following conditions:

$\left(\mathrm{S}_{1}\right) \operatorname{Shift}(\Delta)$ is shifted;

$\left(\mathrm{S}_{2}\right) \operatorname{Shift}(\Delta)=\Delta$ if $\Delta$ is shifted;

$\left(\mathrm{S}_{3}\right) f(\Delta)=f(\operatorname{Shift}(\Delta))$;

$\left(\mathrm{S}_{4}\right) \operatorname{Shift}\left(\Delta^{\prime}\right) \subset \operatorname{Shift}(\Delta)$ if $\Delta^{\prime} \subset \Delta$. 
Erdös, Ko and Rado [5] introduce a combinatorial shifting. Let $\Delta$ be a simplicial complex on $[n]$. Let $1 \leq i<j \leq n$. Write $\operatorname{Shift}_{i j}(\Delta)$ for the simplicial complex on $[n]$ whose faces are $C_{i j}(\sigma) \subset[n]$, where $\sigma \in \Delta$ and where

$$
C_{i j}(\sigma)=\left\{\begin{array}{lc}
(\sigma \backslash\{i\}) \cup\{j\}, & \text { if } i \in \sigma, j \notin \sigma \text { and }(\sigma \backslash\{i\}) \cup\{j\} \notin \Delta, \\
\sigma, & \text { otherwise. }
\end{array}\right.
$$

It follows from, e.g., [7, Corollary 8.6] that there exists a finite sequence of pairs of integers $\left(i_{1}, j_{1}\right),\left(i_{2}, j_{2}\right), \ldots,\left(i_{q}, j_{q}\right)$ with each $1 \leq i_{k}<j_{k} \leq n$ such that

$$
\operatorname{Shift}_{i_{q} j_{q}}\left(\operatorname{Shift}_{i_{q-1} j_{q-1}}\left(\cdots\left(\operatorname{Shift}_{i_{1} j_{1}}(\Delta)\right) \cdots\right)\right)
$$

is shifted. Such a shifted complex is called a combinatorial shifted complex of $\Delta$ and will be denoted by $\Delta^{c}$. A combinatorial shifted complex $\Delta^{c}$ of $\Delta$ is, however, not necessarily unique. The operation $\Delta \mapsto \Delta^{c}$, which is a shifting operation (7, Lemma 8.4]), is called combinatorial shifting.

Assume that the base field $K$ is infinite. The exterior algebraic shifted complex of a simplicial complex $\Delta$ on $[n]$ is the simplicial complex $\Delta^{e}$ on $[n]$ with

$$
J_{\Delta^{e}}=\operatorname{Gin}^{E}\left(J_{\Delta}\right) .
$$

Following [7, p. 105] and [8, p. 125] the operation $\Delta \mapsto \Delta^{e}$, which is a shifting operation ([7, Proposition 8.8]), is called exterior algebraic shifting.

Assume that the base field $K$ is of characteristic 0 . Let $\Delta$ be a simplicial complex on $[n]$ and write $G\left(\operatorname{Gin}^{S}\left(I_{\Delta}\right)\right)$ for the unique minimal system of monomial generators of the generic initial ideal $\operatorname{Gin}^{S}\left(I_{\Delta}\right)$ of the Stanley-Reisner ideal $I_{\Delta}$ of $S$. Let $u=x_{i_{1}} x_{i_{2}} \cdots x_{i_{j}} \cdots x_{i_{s}}$, where $1 \leq i_{1} \leq i_{2} \leq \cdots \leq i_{j} \leq \cdots \leq i_{s} \leq n$, be a monomial belonging to $G\left(\operatorname{Gin}^{S}\left(I_{\Delta}\right)\right)$. One has $i_{s}+(s-1) \leq n$ ([7, Lemma 8.15]). We then introduce the squarefree monomial

$$
u^{*}=x_{i_{1}} x_{i_{2}+1} \cdots x_{i_{j}+(j-1)} \cdots x_{i_{s}+(s-1)}
$$

of $S$ and write $\left(\operatorname{Gin}^{S}\left(I_{\Delta}\right)\right)^{*}$ for the squarefree ideal of $S$ generated by those monomials $u^{*}$ with $u \in G\left(\operatorname{Gin}^{S}\left(I_{\Delta}\right)\right)$. The symmetric algebraic shifted complex of $\Delta$ is the simplicial complex $\Delta^{s}$ on $[n]$ with

$$
I_{\Delta^{s}}=\left(\operatorname{Gin}^{S}\left(I_{\Delta}\right)\right)^{*} .
$$

Since $\operatorname{Gin}^{S}\left(I_{\Delta}\right)$ is strongly stable, it follows that $\Delta^{s}$ is shifted ([7, Lemma 8.17]). The operation $\Delta \mapsto \Delta^{s}$, which is a shifting operation ([7, Theorem 8.19]), is called symmetric algebraic shifting.

\section{Graded Betti numbers of $I_{\Delta^{e}}$ AND $I_{\Delta^{c}}$}

Let $K$ be an infinite field, $S=K\left[x_{1}, \ldots, x_{n}\right]$ the polynomial ring in $n$ variables over $K$ with each $\operatorname{deg} x_{i}=1$ and $E=\bigoplus_{d=0}^{n} \Lambda^{d}(V)$ the exterior algebra of a vector space $V$ over $K$ of dimension $n$ with basis $e_{1}, \ldots, e_{n}$. Assume that the general linear group $\mathrm{GL}(n ; K)$ acts linearly on $E$. Let, as before, $<_{\text {rev }}$ be the reverse lexicographic order on $E$ induced by the ordering $e_{1}>\cdots>e_{n}$.

Given an arbitrary homogeneous ideal $I=\bigoplus_{d=0}^{n} I_{d}$ of $E$ with each $I_{d} \subset \bigwedge^{d}(V)$, fix $\varphi \in \mathrm{GL}(n ; K)$ for which $\operatorname{in}_{<_{\text {rev }}}(\varphi(I))$ is the generic initial ideal $\operatorname{Gin}^{E}(I)$ of $I$. Recall that the subspace $\bigwedge^{d}(V)$ is of dimension $\left(\begin{array}{l}n \\ d\end{array}\right)$ with a canonical $K$-basis $e_{\sigma}$, 


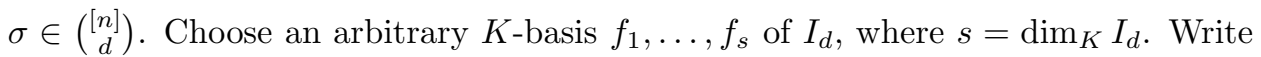
each $\varphi\left(f_{i}\right), 1 \leq i \leq s$, of the form

$$
\varphi\left(f_{i}\right)=\sum_{\sigma \in\left(\begin{array}{c}
{[n]} \\
d
\end{array}\right)} \alpha_{i}^{\sigma} e_{\sigma}
$$

with each $\alpha_{i}^{\sigma} \in K$. Let $M(I, d)$ denote the $s \times\left(\begin{array}{l}n \\ d\end{array}\right)$ matrix

$$
M(I, d)=\left(\alpha_{i}^{\sigma}\right)_{1 \leq i \leq s, \sigma \in\left(\begin{array}{c}
{[n]} \\
d
\end{array}\right)}
$$

whose columns are indexed by $\sigma \in\left(\begin{array}{c}{[n]} \\ d\end{array}\right)$. Moreover, for each $\tau \in\left(\begin{array}{c}{[n]} \\ d\end{array}\right)$, write $M_{\tau}(I, d)$ for the submatrix of $M(I, d)$ which consists of the columns of $M(I, d)$ indexed by those $\sigma \in\left(\begin{array}{c}{[n]} \\ d\end{array}\right)$ with $e_{\tau} \leq_{\text {rev }} e_{\sigma}$ and write $M_{\tau}^{\prime}(I, d)$ for the submatrix of $M_{\tau}(I, d)$ which is obtained by removing the column of $M_{\tau}(I, d)$ indexed by $\tau$.

Lemma 2.1. Let $e_{\tau} \in \bigwedge^{d}(V)$ with $\tau \in\left(\begin{array}{c}{[n]} \\ d\end{array}\right)$. Then one has $e_{\tau} \in\left(\operatorname{Gin}^{E}(I)\right)_{d}$ if and only if $\operatorname{rank}\left(M_{\tau}^{\prime}(I, d)\right)<\operatorname{rank}\left(M_{\tau}(I, d)\right)$.

Proof. In linear algebra we know that $\operatorname{rank}\left(M_{\tau}^{\prime}(I, d)\right)<\operatorname{rank}\left(M_{\tau}(I, d)\right)$ if and only if the row vector $(0, \ldots, 0,1)$ with " 1 " lying on the column indexed by $\tau$ arises in $M_{\tau}(I, d)$ after repeating the elementary transformations on the row vectors of $M_{\tau}(I, d)$. Thus, by identifying the rows of $M(I, d)$ with $\varphi\left(f_{1}\right), \ldots, \varphi\left(f_{s}\right)$, it follows that $\operatorname{rank}\left(M_{\tau}^{\prime}(I, d)\right)<\operatorname{rank}\left(M_{\tau}(I, d)\right)$ if and only if there exist $c_{1}, \ldots, c_{s}$ belonging to $K$ with $\operatorname{in}_{<_{\text {rev }}}(f)=e_{\tau}$, where $f=\sum_{i=1}^{s} c_{i} \varphi\left(f_{i}\right) \in(\varphi(I))_{d}$. Since $\operatorname{Gin}^{E}(I)=\operatorname{in}_{<_{\text {rev }}}(\varphi(I))$, one has $e_{\tau} \in\left(\operatorname{Gin}^{E}(I)\right)_{d}$ if and only if $\operatorname{rank}\left(M_{\tau}^{\prime}(I, d)\right)<$ $\operatorname{rank}\left(M_{\tau}(I, d)\right)$, as desired.

Corollary 2.2. The rank of a matrix $M_{\tau}(I, d), \tau \in\left(\begin{array}{c}{[n]} \\ d\end{array}\right)$, is independent of the choice of $\varphi \in \operatorname{GL}(n ; K)$ for which $\operatorname{Gin}^{E}(I)=\operatorname{in}_{<_{\text {rev }}}(\varphi(I))$ together with a $K$-basis $f_{1}, \ldots, f_{s}$ of $I_{d}$.

Corollary 2.3. Let $I \subset E$ be a homogeneous ideal and $\psi \in \mathrm{GL}(n ; K)$. Then one has $\operatorname{rank}\left(M_{\tau}(I, d)\right)=\operatorname{rank}\left(M_{\tau}(\psi(I), d)\right)$ for all $\tau \in\left(\begin{array}{c}{[n]} \\ d\end{array}\right)$.

Proof. Recall that there is a nonempty subset $U \subset \mathrm{GL}(n ; K)$ which is Zariski open and dense such that $\operatorname{Gin}^{E}(I)=\operatorname{in}_{<_{\text {rev }}}(\varphi(I))$ for all $\varphi \in U$. Similarly, there is a nonempty subset $V \subset \mathrm{GL}(n ; K)$ which is Zariski open and dense such that $\operatorname{Gin}^{E}(\psi(I))=\operatorname{in}_{<_{\text {rev }}}\left(\varphi^{\prime}(\psi(I))\right)$ for all $\varphi^{\prime} \in V$. Since $U \psi^{-1} \cap V \neq \emptyset$, if $\rho \in U \psi^{-1} \cap V$, then $\operatorname{Gin}^{E}(I)=\operatorname{in}_{<_{\text {rev }}}(\rho(\psi(I)))=\operatorname{Gin}^{E}(\psi(I))$ and the matrix $M(I, d)$ using $\rho \psi \in$ $U$ and a $K$-basis $f_{1}, \ldots, f_{s}$ of $I_{d}$ coincides with $M(\psi(I), d)$ using $\rho \in V$ and a $K$-basis $\psi\left(f_{1}\right), \ldots, \psi\left(f_{s}\right)$ of $\psi(I)_{d}$.

If $u=e_{\sigma}$ is a monomial of $E$, then we set $m(u)=\max \{j: j \in \sigma\}$. Given a monomial ideal $I \subset E$, one defines $m_{\leq i}(I, d)$, where $1 \leq i \leq n$ and $1 \leq d \leq n$, by

$$
m_{\leq i}(I, d)=\left|\left\{u=e_{\sigma} \in I: \operatorname{deg}(u)=d, m(u) \leq i\right\}\right| .
$$

Corollary 2.4. Let $\sigma_{(i, d)}=\{i-d+1, i-d+2, \ldots, i\} \in\left(\begin{array}{c}{[n]} \\ d\end{array}\right)$. Then given a homogeneous ideal $I \subset E$ one has

$$
m_{\leq i}\left(\operatorname{Gin}^{E}(I), d\right)=\operatorname{rank}\left(M_{\sigma_{(i, d)}}(I, d)\right),
$$

where $\operatorname{rank}\left(M_{\sigma_{(i, d)}}(I, d)\right)=0$ if $i<d$. 
Proof. Let $\tau \in\left(\begin{array}{c}{[n]} \\ d\end{array}\right)$. Then $m\left(e_{\tau}\right) \leq i$ if and only if $e_{\sigma_{(i, d)}} \leq_{\text {rev }} e_{\tau}$. On the other hand, Lemma 2.1 says that $\operatorname{rank}\left(M_{\sigma_{(i, d)}}(I, d)\right)$ coincides with the number of monomials $e_{\tau} \in\left(\operatorname{Gin}^{E}(I)\right)_{d}$ with $e_{\sigma_{(i, d)}} \leq_{\text {rev }} e_{\tau}$. Thus $m_{\leq i}\left(\operatorname{Gin}^{E}(I), d\right)=$ $\operatorname{rank}\left(M_{\sigma_{(i, d)}}(I, d)\right)$, as required.

Let $I \subset E$ be a monomial ideal. Fix $1 \leq i<j \leq n$. Let $t \in K$ and introduce the linear injective map $S_{i j}^{t}: I \rightarrow E$ satisfying

$$
S_{i j}^{t}\left(e_{\sigma}\right)= \begin{cases}e_{(\sigma \backslash\{j\}) \cup\{i\}}+t e_{\sigma}, & \text { if } j \in \sigma, i \notin \sigma \text { and } e_{(\sigma \backslash\{j\}) \cup\{i\}} \notin I, \\ e_{\sigma}, & \text { otherwise, }\end{cases}
$$

where $e_{\sigma} \in I$ is a monomial. Let $I_{i j}(t) \subset E$ denote the image of $I$ by $S_{i j}^{t}$.

Lemma 2.5. (a) If $t \neq 0$, then there is $\lambda_{i j}^{t} \in \mathrm{GL}(n ; K)$ with $I_{i j}(t)=\lambda_{i j}^{t}(I)$. In particular the subspace $I_{i j}(t)$ is an ideal of $E$.

(b) Let $\Delta$ denote the simplicial complex on $[n]$ and $J_{\Delta}$ its exterior face ideal. Then $\left(J_{\Delta}\right)_{i j}(0)=J_{\operatorname{Shift}_{i j}(\Delta)}$.

Proof. (a) Let $\lambda_{i j}^{t} \in \mathrm{GL}(n ; K)$ satisfy

$$
\lambda_{i j}^{t}\left(e_{k}\right)= \begin{cases}e_{k} & (k \neq j), \\ e_{i}+t e_{j} & (k=j) .\end{cases}
$$

We claim $I_{i j}(t)=\lambda_{i j}^{t}(I)$. Let $e_{\sigma} \in I$.

(i) If $j \notin \sigma$, then $\lambda_{i j}^{t}\left(e_{\sigma}\right)=e_{\sigma}=S_{i j}^{t}\left(e_{\sigma}\right)$. Thus $\lambda_{i j}^{t}\left(e_{\sigma}\right) \in I_{i j}(t)$.

(ii) If $j \in \sigma$ and $i \in \sigma$, then $\lambda_{i j}^{t}\left(e_{\sigma}\right)=t e_{\sigma}=t S_{i j}^{t}\left(e_{\sigma}\right)$. Thus $\lambda_{i j}^{t}\left(e_{\sigma}\right) \in I_{i j}(t)$.

(iii) Let $j \in \sigma$ and $i \notin \sigma$ with $e_{(\sigma \backslash\{j\}) \cup\{i\}} \in I$. Then $\lambda_{i j}^{t}\left(e_{\sigma}\right)=e_{(\sigma \backslash\{j\}) \cup\{i\}}+t e_{\sigma}$ and $S_{i j}^{t}\left(e_{\sigma}\right)=e_{\sigma}$. Since $e_{(\sigma \backslash\{j\}) \cup\{i\}} \in I, S_{i j}^{t}\left(e_{(\sigma \backslash\{j\}) \cup\{i\}}\right)=e_{(\sigma \backslash\{j\}) \cup\{i\}} \in$ $I_{i j}(t)$. Thus $\lambda_{i j}^{t}\left(e_{\sigma}\right) \in I_{i j}(t)$.

(iv) Let $j \in \sigma$ and $i \notin \sigma$ with $e_{(\sigma \backslash\{j\}) \cup\{i\}} \notin I$. Then $\lambda_{i j}^{t}\left(e_{\sigma}\right)=e_{(\sigma \backslash\{j\}) \cup\{i\}}+t e_{\sigma}$ and $S_{i j}^{t}\left(e_{\sigma}\right)=e_{(\sigma \backslash\{j\}) \cup\{i\}}+t e_{\sigma}$. Thus $\lambda_{i j}^{t}\left(e_{\sigma}\right) \in I_{i j}(t)$.

Hence $\lambda_{i j}^{t}(I) \subset I_{i j}(t)$. Since each of $\lambda_{i j}^{t}$ and $S_{i j}^{t}$ is injective, one has $I_{i j}(t)=\lambda_{i j}^{t}(I)$, as desired.

(b) We claim $\left\{\sigma \subset[n]: e_{\sigma} \in\left(J_{\Delta}\right)_{i j}(0)\right\} \cap \operatorname{Shift}_{i j}(\Delta)=\emptyset$.

(i) If $e_{\sigma} \in\left(J_{\Delta}\right)_{i j}(0)$ with $e_{\sigma} \notin J_{\Delta}$, then there is $e_{\tau} \in J_{\Delta}$ with $\sigma=(\tau \backslash\{j\})$ $\cup\{i\}$. Since $\sigma \in \Delta, \tau \notin \Delta$ and $\tau=(\sigma \backslash\{i\}) \cup\{j\}$, one has $\tau=C_{i j}(\sigma) \in$ $\operatorname{Shift}_{i j}(\Delta)$. Thus $\sigma \notin \operatorname{Shift}_{i j}(\Delta)$.

(ii) Let $e_{\sigma} \in\left(J_{\Delta}\right)_{i j}(0)$ with $e_{\sigma} \in J_{\Delta}$. Suppose $\sigma \in \operatorname{Shift}_{i j}(\Delta)$. Since $\sigma \notin \Delta$, there is $\tau \subset[n]$ with $\tau \in \Delta$ such that $\sigma=(\tau \backslash\{i\}) \cup\{j\}$. Hence $j \in \sigma$, $i \notin \sigma$ and $e_{\tau}=e_{(\sigma \backslash\{j\}) \cup\{i\}} \notin J_{\Delta}$. Thus $e_{\tau} \in\left(J_{\Delta}\right)_{i j}(0)$ and $e_{\sigma} \notin\left(J_{\Delta}\right)_{i j}(0)$.

Hence $\left(J_{\Delta}\right)_{i j}(0) \subset J_{\operatorname{Shift}_{i j}(\Delta)}$. Since $\operatorname{dim}_{K}\left(J_{\Delta}\right)_{i j}(0)=\operatorname{dim}_{K} J_{\Delta}=\operatorname{dim}_{K} J_{\operatorname{Shift}_{i j}(\Delta)}$, it follows that $\left(J_{\Delta}\right)_{i j}(0)=J_{\operatorname{Shift}_{i j}(\Delta)}$.

Lemma 2.6. Work with the same notation as in Corollary 2.4, One has

$$
\operatorname{rank}\left(M_{\sigma_{(i, d)}}\left(J_{\operatorname{Shift}_{i j}(\Delta)}, d\right)\right) \leq \operatorname{rank}\left(M_{\sigma_{(i, d)}}\left(J_{\Delta}, d\right)\right) .
$$

Proof. Fix a finite set $A \subset K$ with $0 \in A$ for which $|A| \geq\left(\begin{array}{l}n \\ d\end{array}\right)+2$. One has $\varphi \in \operatorname{GL}(n ; K)$ for which $\operatorname{in}_{<_{\text {rev }}}\left(\varphi\left(\left(J_{\Delta}\right)_{i j}(t)\right)\right)$ is the generic initial ideal of $\left(J_{\Delta}\right)_{i j}(t)$ for all $t \in A$. For each $\sigma \in\left(\begin{array}{c}{[n]} \\ d\end{array}\right)$ we write

$$
\varphi\left(e_{\sigma}\right)=\sum_{\tau \in\left(\begin{array}{c}
{[n]} \\
d
\end{array}\right)} c_{\sigma}^{\tau} e_{\tau}, \quad c_{\sigma}^{\tau} \in K
$$


By using $\varphi$ together with the $K$-basis $\left\{S_{i j}^{t}\left(e_{\sigma}\right): e_{\sigma} \in\left(J_{\Delta}\right)_{d}\right\}$ of $\left(\left(J_{\Delta}\right)_{i j}(t)\right)_{d}$, we compute the matrix $M\left(\left(J_{\Delta}\right)_{i j}(t), d\right)$. If $S_{i j}^{t}\left(e_{\sigma}\right)=e_{(\sigma \backslash\{j\}) \cup\{i\}}+t e_{\sigma}$, then

$$
\varphi\left(S_{i j}^{t}\left(e_{\sigma}\right)\right)=\sum_{\tau \in\left(\begin{array}{c}
{[n]} \\
d
\end{array}\right)}\left(c_{(\sigma \backslash\{j\}) \cup\{i\}}^{\tau}+t c_{\sigma}^{\tau}\right) e_{\tau} .
$$

Hence

$$
M\left(\left(J_{\Delta}\right)_{i j}(t), d\right)=\left(\alpha_{\ell}^{\sigma}+t \beta_{\ell}^{\sigma}\right)_{1 \leq \ell \leq \operatorname{dim}_{K}\left(\left(J_{\Delta}\right)_{i j}(t)\right)_{d}, \sigma \in\left(\begin{array}{c}
{[n]} \\
d
\end{array}\right)}
$$

with each $\alpha_{\ell}^{\sigma}, \beta_{\ell}^{\sigma} \in K$.

Let $r(t)=\operatorname{rank}\left(M_{\sigma_{(i, d)}}\left(\left(J_{\Delta}\right)_{i j}(t), d\right)\right)$. Thus $r(t)$ coincides with the largest size of nonzero minors of the matrix $M_{\sigma_{(i, d)}}\left(\left(J_{\Delta}\right)_{i j}(t), d\right)$. Fix a minor $N(t)$ of size $r(0)$ of $M_{\sigma_{(i, d)}}\left(\left(J_{\Delta}\right)_{i j}(t), d\right)$ with $N(0) \neq 0$. We regard $N(t)$ as a polynomial in $t$ of degree at most $r(0)$. Since $r(0) \leq\left(\begin{array}{l}n \\ d\end{array}\right)$ and $|A| \geq\left(\begin{array}{l}n \\ d\end{array}\right)+2$, it follows that there is $0 \neq a \in A$ with $N(a) \neq 0$. Hence $r(0) \leq r(a)$. Corollary 2.3 together with Lemma 2.5 now guarantees that $r(0)=\operatorname{rank}\left(M_{\sigma_{(i, d)}}\left(J_{\operatorname{Shift}_{i j}(\Delta)}, d\right)\right)$ and $r(a)=\operatorname{rank}\left(M_{\sigma_{(i, d)}}\left(J_{\Delta}, d\right)\right)$. $\operatorname{Thus} \operatorname{rank}\left(M_{\sigma_{(i, d)}}\left(J_{\operatorname{Shift}_{i j}(\Delta)}, d\right)\right) \leq \operatorname{rank}\left(M_{\sigma_{(i, d)}}\left(J_{\Delta}, d\right)\right)$, as desired.

Corollary 2.7. Let $\Delta$ be a simplicial complex on $[n]$. Then for all $i$ and $d$ one has

$$
m_{\leq i}\left(J_{\Delta^{e}}, d\right) \geq m_{\leq i}\left(J_{\Delta^{c}}, d\right) .
$$

Proof. Corollary 2.4 together with Lemma 2.6 guarantees that

$$
m_{\leq i}\left(\operatorname{Gin}^{E}\left(J_{\Delta}\right), d\right) \geq m_{\leq i}\left(\operatorname{Gin}^{E}\left(J_{\operatorname{Shift}_{i j}(\Delta)}\right), d\right) .
$$

Hence $m_{\leq i}\left(\operatorname{Gin}^{E}\left(J_{\Delta}\right), d\right) \geq m_{\leq i}\left(\operatorname{Gin}^{E}\left(J_{\Delta^{c}}\right), d\right)$. In other words, one has $m_{\leq i}\left(J_{\Delta^{e}}, d\right)$ $\geq m_{\leq i}\left(J_{\left(\Delta^{c}\right)^{e}}, d\right)$. However, since $\Delta^{c}$ is shifted, it follows that $\left(\Delta^{c}\right)^{e}=\bar{\Delta}^{c}$. Thus $m_{\leq i}\left(J_{\Delta^{e}}, d\right) \geq m_{\leq i}\left(J_{\Delta^{c}}, d\right)$, as desired.

We now approach the final step to prove the inequalities $\beta_{i i+j}\left(I_{\Delta^{e}}\right) \leq \beta_{i i+j}\left(I_{\Delta^{c}}\right)$ for all $i$ and $j$ on graded Betti numbers of $I_{\Delta^{e}}$ and $I_{\Delta^{c}}$. Lemma 2.8 stated below essentially appears in [1, pp. $376-377]$.

Lemma 2.8. If $\Delta$ is a shifted simplicial complex, then for all $i$ and $j$ one has

$$
\begin{aligned}
\beta_{i i+j}\left(I_{\Delta}\right)= & m_{\leq n}\left(J_{\Delta}, j\right)\left(\begin{array}{c}
n-j \\
i
\end{array}\right)-\sum_{k=j}^{n-1} m_{\leq k}\left(J_{\Delta}, j\right)\left(\begin{array}{c}
k-j \\
i-1
\end{array}\right) \\
& -\sum_{k=j}^{n} m_{\leq k-1}\left(J_{\Delta}, j-1\right)\left(\begin{array}{c}
k-j \\
i
\end{array}\right) .
\end{aligned}
$$

Corollary 2.9. Let $\Delta$ and $\Delta^{\prime}$ be shifted simplicial complexes on $[n]$ with $f(\Delta)=$ $f\left(\Delta^{\prime}\right)$ and suppose that

$$
m_{\leq i}\left(J_{\Delta}, j\right) \geq m_{\leq i}\left(J_{\Delta^{\prime}}, j\right)
$$

for all $i$ and $j$. Then for all $i$ and $j$ one has

$$
\beta_{i i+j}\left(I_{\Delta}\right) \leq \beta_{i i+j}\left(I_{\Delta^{\prime}}\right) .
$$

Proof. Since $f(\Delta)=f\left(\Delta^{\prime}\right)$, one has $m_{\leq n}\left(J_{\Delta}, j\right)=m_{\leq n}\left(J_{\Delta^{\prime}}, j\right)$ for all $j$. Lemma 2.8 then yields the inequalities $\beta_{i i+j}\left(I_{\Delta}\right) \leq \beta_{i i+j}\left(I_{\Delta^{\prime}}\right)$ for all $i$ and $j$, as desired. 
Theorem 2.10. Let the base field be infinite. Let $\Delta$ be a simplicial complex, $\Delta^{e}$ the exterior algebraic shifted complex of $\Delta$ and $\Delta^{c}$ a combinatorial shifted complex of $\Delta$. Then

for all $i$ and $j$.

$$
\beta_{i i+j}\left(I_{\Delta^{e}}\right) \leq \beta_{i i+j}\left(I_{\Delta^{c}}\right)
$$

Proof. Corollary 2.7 guarantees $m_{\leq i}\left(J_{\Delta^{c}}, j\right) \leq m_{\leq i}\left(J_{\Delta^{e}}, j\right)$ for all $i$ and $j$. Thus by virtue of Corollary 2.9 the required inequalities $\beta_{i i+j}\left(I_{\Delta^{e}}\right) \leq \beta_{i i+j}\left(I_{\Delta^{c}}\right)$ follow immediately.

\section{Graded Betti numbers of $I_{\Delta}$ And $I_{\Delta^{c}}$}

Let $K$ be an arbitrary field, and let $S=K\left[x_{1}, \ldots, x_{n}\right]$ denote the polynomial ring in $n$ variables over $K$ with each $\operatorname{deg} x_{i}=1$. Let $\Delta$ be a simplicial complex on $[n]$ and $I_{\Delta} \subset S$ its Stanley-Reisner ideal. Let $\tilde{H}_{k}(\Delta ; K)$ denote the $k$ th reduced homology group of $\Delta$ with coefficients $K$. If $W \subset[n]$, then $\Delta_{W}$ stands for the simplicial complex on $W$ whose faces are those faces $\sigma$ of $\Delta$ with $\sigma \subset W$.

Recall that Hochster's formula [4, Theorem 5.5.1] to compute the graded Betti numbers of $I_{\Delta}$ says that

$$
\beta_{i i+j}\left(I_{\Delta}\right)=\sum_{W \subset[n],|W|=i+j} \operatorname{dim}_{K}\left(\tilde{H}_{j-2}\left(\Delta_{W} ; K\right)\right)
$$

for all $i$ and $j$.

Fix $1 \leq i<j \leq n$ and set $\Gamma=\operatorname{Shift}_{i j}(\Delta)$.

Lemma 3.1. One has

$$
\operatorname{dim}_{K}\left(\tilde{H}_{k}(\Delta ; K)\right) \leq \operatorname{dim}_{K}\left(\tilde{H}_{k}(\Gamma ; K)\right)
$$

for all $k$.

Proof. By considering an extension field of $K$ if necessary, we assume that $K$ is infinite. Let $\Delta^{e}$ denote the exterior algebraic shifted complex of $\Delta$. It is known [7. Proposition 8.10] that $\tilde{H}_{k}(\Delta ; K) \cong \tilde{H}_{k}\left(\Delta^{e} ; K\right)$. Thus what we must prove is $\operatorname{dim}_{K}\left(\tilde{H}_{k}\left(\Delta^{e} ; K\right)\right) \leq \operatorname{dim}_{K}\left(\tilde{H}_{k}\left(\Gamma^{e} ; K\right)\right)$ for all $k$. By using $(2)$ one has $\beta_{i n}\left(I_{\Delta}\right)=$ $\operatorname{dim}_{K}\left(\tilde{H}_{n-i-2}(\Delta ; K)\right)$. Hence our work is to show that $\beta_{i n}\left(I_{\Delta^{e}}\right) \leq \beta_{i n}\left(I_{\Gamma^{e}}\right)$ for all $i$. The inequality (1) says that $m_{\leq i}\left(J_{\Delta^{e}}, j\right) \geq m_{\leq i}\left(J_{\Gamma^{e}}, j\right)$ for all $i$ and $j$. It then follows from Corollary 2.9 that $\beta_{i i+j}\left(I_{\Delta^{e}}\right) \leq \beta_{i i+j}\left(I_{\Gamma^{e}}\right)$ for all $i$ and $j$. Thus in particular $\beta_{i n}\left(I_{\Delta^{e}}\right) \leq \beta_{i n}\left(I_{\Gamma^{e}}\right)$ for all $i$.

Let $W \subset[n] \backslash\{i, j\}$. Let $\Delta_{1}=\Delta_{W \cup\{i\}}, \Delta_{2}=\Delta_{W \cup\{j\}}, \Gamma_{1}=\Gamma_{W \cup\{i\}}$ and $\Gamma_{2}=\Gamma_{W \cup\{j\}}$. Then

$$
\begin{gathered}
\Delta_{1} \cap \Delta_{2}=\Gamma_{1} \cap \Gamma_{2}=\Delta_{W}=\Gamma_{W}, \\
\Gamma_{1} \cup \Gamma_{2}=\operatorname{Shift}_{i j}\left(\Delta_{1} \cup \Delta_{2}\right) .
\end{gathered}
$$

Recall that the reduced Mayer-Vietoris exact sequence of $\Delta_{1}$ and $\Delta_{2}$ and that of $\Gamma_{1}$ and $\Gamma_{2}$ are the exact sequences

$$
\begin{aligned}
& \cdots \longrightarrow \tilde{H}_{k}\left(\Delta_{W} ; K\right) \stackrel{\partial_{1, k}}{\longrightarrow} \tilde{H}_{k}\left(\Delta_{1} ; K\right) \oplus \tilde{H}_{k}\left(\Delta_{2} ; K\right) \stackrel{\partial_{2, k}}{\longrightarrow} \tilde{H}_{k}\left(\Delta_{1} \cup \Delta_{2} ; K\right) \\
& \stackrel{\partial_{3, k}}{\longrightarrow} \tilde{H}_{k-1}\left(\Delta_{W} ; K\right) \stackrel{\partial_{1, k-1}}{\longrightarrow} \cdots, \\
& \cdots \longrightarrow \tilde{H}_{k}\left(\Gamma_{W} ; K\right) \stackrel{\partial_{1, k}^{\prime}}{\longrightarrow} \tilde{H}_{k}\left(\Gamma_{1} ; K\right) \oplus \tilde{H}_{k}\left(\Gamma_{2} ; K\right) \stackrel{\partial_{2, k}^{\prime}}{\longrightarrow} \tilde{H}_{k}\left(\Gamma_{1} \cup \Gamma_{2} ; K\right) \\
& \stackrel{\partial_{3, k}^{\prime}}{\longrightarrow} \tilde{H}_{k-1}\left(\Gamma_{W} ; K\right) \stackrel{\partial_{1, k-1}^{\prime}}{\longrightarrow} \cdots .
\end{aligned}
$$


Lemma 3.2. One has

for all $k$.

$$
\operatorname{Ker}\left(\partial_{1, k}^{\prime}\right) \subset \operatorname{Ker}\left(\partial_{1, k}\right)
$$

Proof. Let $\pi$ be a permutation on $[n]$ with $\pi(i)<\pi(j)$ and $\pi(\Delta)$ the simplicial complex $\{\pi(F): F \in \Delta\}$ on $[n]$. Since the combinatorial type of $\operatorname{Shift}_{i j}(\Delta)$ is equal to that of $\operatorname{Shift}_{\pi(i) \pi(j)}(\pi(\Delta))$, we will assume that $j=i+1$.

Let, in general, $C_{k}(\Delta)$ denote the vector space over $K$ with basis $\left\{e_{i_{0} i_{1} \cdots i_{k}}\right\}$, where $\left\{i_{0}, i_{1}, \ldots, i_{k}\right\} \in \Delta$ and where $1 \leq i_{0}<i_{1}<\cdots<i_{k} \leq n$, and define the linear map $\partial: C_{k}(\Delta) \rightarrow C_{k-1}(\Delta)$ by setting $\partial\left(e_{i_{0} i_{1} \cdots i_{k}}\right)=\sum_{j=0}^{k}(-1)^{j} e_{i_{0} i_{1} \cdots i_{j-1} i_{j+1} \cdots i_{k}}$.

Let $[a] \in \operatorname{Ker}\left(\partial_{1, k}^{\prime}\right)$, where $a \in C_{k}\left(\Gamma_{W}\right)$. Since $([a],[a]) \in \tilde{H}_{k}\left(\Gamma_{1} ; K\right) \oplus \tilde{H}_{k}\left(\Gamma_{2} ; K\right)$ vanishes, one has $u \in C_{k+1}\left(\Gamma_{1}\right)$ with $\partial(u)=a$. Say,

$$
u=\sum_{|F|=k+1, i \notin F, F \cup\{i\} \in \Gamma_{1}} a_{F \cup\{i\}} e_{F \cup\{i\}}+\sum_{|G|=k+2, G \in \Delta_{W}} b_{G} e_{G},
$$

where $a_{F \cup\{i\}}, b_{G} \in K$.

Let $F \subset W$ with $F \cup\{i\} \in \Gamma_{1}$. Then $F \cup\{i\} \in \Delta_{1}$ and $F \cup\{j\} \in \Delta_{2}$. Thus $F \cup\{j\} \in \Gamma_{2}$. In particular $u \in C_{k+1}\left(\Delta_{1}\right)$ with $\partial(u)=a$.

Since $a \in C_{k}\left(\Gamma_{W}\right)$ is a linear combination of those basis elements $e_{F}$ with $F \in \Gamma$, $F \subset W$ and $|F|=k+1$ and since $j=i+1$, it follows that $\partial(v)=a$, where $v \in C_{k+1}\left(\Gamma_{2}\right) \cap C_{k+1}\left(\Delta_{2}\right)$ is the element

$$
v=\sum_{|F|=k+1, i \notin F, F \cup\{i\} \in \Gamma_{1}} a_{F \cup\{i\}} e_{F \cup\{j\}}+\sum_{|G|=k+2, G \in \Delta_{W}} b_{G} e_{G} .
$$

Hence $([a],[a]) \in \tilde{H}_{k}\left(\Delta_{1} ; K\right) \oplus \tilde{H}_{k}\left(\Delta_{2} ; K\right)$ vanishes, as required.

It then follows that

$$
\begin{gathered}
\operatorname{dim}_{K}\left(\operatorname{Ker}\left(\partial_{1, k}\right)\right) \geq \operatorname{dim}_{K}\left(\operatorname{Ker}\left(\partial_{1, k}^{\prime}\right)\right), \\
\operatorname{dim}_{K}\left(\operatorname{Im}\left(\partial_{1, k}\right)\right) \leq \operatorname{dim}_{K}\left(\operatorname{Im}\left(\partial_{1, k}^{\prime}\right)\right), \\
\operatorname{dim}_{K}\left(\operatorname{Ker}\left(\partial_{2, k}\right)\right) \leq \operatorname{dim}_{K}\left(\operatorname{Ker}\left(\partial_{2, k}^{\prime}\right)\right) .
\end{gathered}
$$

On the other hand,

$$
\begin{gathered}
\operatorname{dim}_{K}\left(\tilde{H}_{k}\left(\Delta_{1} \cup \Delta_{2} ; K\right)\right)=\operatorname{dim}_{K}\left(\operatorname{Ker}\left(\partial_{3, k}\right)\right)+\operatorname{dim}_{K}\left(\operatorname{Im}\left(\partial_{3, k}\right)\right), \\
\operatorname{dim}_{K}\left(\tilde{H}_{k}\left(\Gamma_{1} \cup \Gamma_{2} ; K\right)\right)=\operatorname{dim}_{K}\left(\operatorname{Ker}\left(\partial_{3, k}^{\prime}\right)\right)+\operatorname{dim}_{K}\left(\operatorname{Im}\left(\partial_{3, k}^{\prime}\right)\right) .
\end{gathered}
$$

Lemma 3.1 together with (3) guarantees that

$$
\operatorname{dim}_{K}\left(\tilde{H}_{k}\left(\Delta_{1} \cup \Delta_{2} ; K\right)\right) \leq \operatorname{dim}_{K}\left(\tilde{H}_{k}\left(\Gamma_{1} \cup \Gamma_{2} ; K\right)\right) .
$$

Since $\operatorname{Im}\left(\partial_{3, k}\right)=\operatorname{Ker}\left(\partial_{1, k-1}\right)$ and $\operatorname{Im}\left(\partial_{3, k}^{\prime}\right)=\operatorname{Ker}\left(\partial_{1, k-1}^{\prime}\right)$, Lemma 3.2 yields

$$
\operatorname{dim}_{K}\left(\operatorname{Im}\left(\partial_{3, k}\right)\right) \geq \operatorname{dim}_{K}\left(\operatorname{Im}\left(\partial_{3, k}^{\prime}\right)\right) .
$$

Since $\operatorname{Im}\left(\partial_{2, k}\right)=\operatorname{Ker}\left(\partial_{3, k}\right)$ and $\operatorname{Im}\left(\partial_{2, k}^{\prime}\right)=\operatorname{Ker}\left(\partial_{3, k}^{\prime}\right)$, it follows from (5) and (6) together with (7) and (8) that

$$
\operatorname{dim}_{K}\left(\operatorname{Im}\left(\partial_{2, k}\right)\right) \leq \operatorname{dim}_{K}\left(\operatorname{Im}\left(\partial_{2, k}^{\prime}\right)\right) .
$$

Finally, it follows from the reduced Mayer-Vietoris exact sequence of $\Delta_{1}$ and $\Delta_{2}$ and that of $\Gamma_{1}$ and $\Gamma_{2}$ together with (4) and (9) that

$$
\operatorname{dim}_{K}\left(\tilde{H}_{k}\left(\Delta_{1} ; K\right) \oplus \tilde{H}_{k}\left(\Delta_{2} ; K\right)\right) \leq \operatorname{dim}_{K}\left(\tilde{H}_{k}\left(\Gamma_{1} ; K\right) \oplus \tilde{H}_{k}\left(\Gamma_{2} ; K\right)\right) .
$$


Lemma 3.3. Fix $1 \leq p<q \leq n$. Let $\Delta$ be a simplicial complex on $[n]$ and $\Gamma=\operatorname{Shift}_{p q}(\Delta)$. Then

$$
\beta_{i i+j}\left(I_{\Delta}\right) \leq \beta_{i i+j}\left(I_{\Gamma}\right)
$$

for all $i$ and $j$.

Proof. The right-hand side of Hochster's formula (11) can be rewritten as

$$
\beta_{i i+j}\left(I_{\Delta}\right)=\alpha_{i j}(\Delta)+\gamma_{i j}(\Delta)+\delta_{i j}(\Delta),
$$

where

$$
\begin{aligned}
\alpha_{i j}(\Delta)= & \sum_{W \subset[n] \backslash\{p, q\},|W|=i+j} \operatorname{dim}_{K}\left(\tilde{H}_{j-2}\left(\Delta_{W} ; K\right)\right), \\
\gamma_{i j}(\Delta)= & \sum_{W \subset[n] \backslash\{p, q\},|W|=i+j-1} \operatorname{dim}_{K}\left(\tilde{H}_{j-2}\left(\Delta_{W \cup\{p\}} ; K\right)\right) \\
& +\sum_{W \subset[n] \backslash\{p, q\},|W|=i+j-1} \operatorname{dim}_{K}\left(\tilde{H}_{j-2}\left(\Delta_{W \cup\{q\}} ; K\right)\right), \\
\delta_{i j}(\Delta)= & \sum_{W \subset[n] \backslash\{p, q\},|W|=i+j-2} \operatorname{dim}_{K}\left(\tilde{H}_{j-2}\left(\Delta_{W \cup\{p, q\}} ; K\right)\right) .
\end{aligned}
$$

Let $W \subset[n] \backslash\{p, q\}$. Then $\Delta_{W}=\Gamma_{W}$. Thus $\alpha_{i j}(\Delta)=\alpha_{i j}(\Gamma)$. Since $\Gamma_{W \cup\{p, q\}}=$ $\operatorname{Shift}\left(\Delta_{W \cup\{p, q\}}\right)$, Lemma 3.1 says that $\delta_{i j}(\Delta) \leq \delta_{i j}(\Gamma)$. Finally, it follows from (10) that $\gamma_{i j}(\Delta) \leq \gamma_{i j}(\Gamma)$. Hence $\beta_{i i+j}\left(I_{\Delta}\right) \leq \beta_{i i+j}\left(I_{\Gamma}\right)$, as desired.

Lemma 3.3 together with the definition of combinatorial shifting now guarantees that

Theorem 3.4. Let the base field be arbitrary. Let $\Delta$ be a simplicial complex and $\Delta^{c}$ a combinatorial shifted complexes of $\Delta$. Then

$$
\beta_{i i+j}\left(I_{\Delta}\right) \leq \beta_{i i+j}\left(I_{\Delta^{c}}\right)
$$

for all $i$ and $j$.

Let $\Delta^{\prime}$ be a shifted simplicial complex with the same $f$-vector as $\Delta$ and $\Delta^{\text {lex }}$ the unique lexsegment simplicial complex with the same $f$-vector as $\Delta$. It is known 1 , Theorem 4.4] that $\beta_{i i+j}\left(I_{\Delta^{\prime}}\right) \leq \beta_{i i+j}\left(I_{\Delta^{\mathrm{lex}}}\right)$ for all $i$ and $j$. Since $\Delta^{c}$ is shifted with $f\left(\Delta^{c}\right)=f(\Delta)$, it follows that $\beta_{i i+j}\left(I_{\Delta^{c}}\right) \leq \beta_{i i+j}\left(I_{\Delta^{\operatorname{lex}}}\right)$ for all $i$ and $j$. Hence

Corollary 3.5. Let the base field be arbitrary. Let $\Delta$ be a simplicial complex and $\Delta^{\text {lex }}$ the unique lexsegment simplicial complex with the same $f$-vector as $\Delta$. Then

$$
\beta_{i i+j}\left(I_{\Delta}\right) \leq \beta_{i i+j}\left(I_{\Delta^{\text {lex }}}\right)
$$

for all $i$ and $j$.

\section{BAD BEHAVIOR OF COMBINATORIAL SHIFTED COMPLEXES}

Given a simplicial complex $\Delta$, do there exist combinatorial shifted complexes $\Delta_{b}^{c}$ and $\Delta_{\sharp}^{c}$ of $\Delta$ such that, for all combinatorial shifted complexes $\Delta^{c}$ of $\Delta$ and for all $i$ and $j$, one has

$$
\beta_{i i+j}\left(I_{\Delta_{b}^{c}}\right) \leq \beta_{i i+j}\left(I_{\Delta^{c}}\right) \leq \beta_{i i+j}\left(I_{\Delta_{\sharp}^{c}}\right) ?
$$

Unfortunately, in general, the existence of such combinatorial shifted complexes $\Delta_{b}^{c}$ and $\Delta_{\sharp}^{c}$ cannot be expected. 
Let $V$ be a vector space of dimension 15 with basis $e_{1}, \ldots, e_{15}$ and $E=$ $\bigoplus_{d=0}^{15} \bigwedge^{d}(V)$ the exterior algebra of $V$. Let $<_{\text {lex }}$ denote the lexicographic order on $E$ induced by the ordering $e_{1}>\cdots>e_{15}$. To simplify the notation we employ the following:

$$
\begin{gathered}
h_{1}=e_{1}, h_{2}=e_{2} \wedge e_{3}, h_{3}=e_{3} \wedge e_{4} \wedge e_{5} \\
h_{4}=e_{4} \wedge \cdots \wedge e_{7}, h_{5}=e_{5} \wedge \cdots \wedge e_{9}, h_{6}=e_{6} \wedge \cdots \wedge e_{11} .
\end{gathered}
$$

First, we introduce $H_{i} \subset \bigwedge^{2}(V)$ with $3 \leq i \leq 8$ and $A, B \subset \bigwedge^{2}(V)$ by setting

$$
\begin{array}{rlrl}
H_{3} & =\left\{e_{12} \wedge e_{13}, e_{12} \wedge e_{15}, e_{13} \wedge e_{14}\right\}, & H_{4}=\left\{e_{12} \wedge e_{13}, e_{12} \wedge e_{14}, e_{14} \wedge e_{15}\right\}, \\
H_{5}=\left\{e_{12} \wedge e_{13}, e_{12} \wedge e_{15}, e_{14} \wedge e_{15}\right\}, & H_{6}=\left\{e_{12} \wedge e_{13}, e_{13} \wedge e_{14}, e_{14} \wedge e_{15}\right\}, \\
H_{7}=\left\{e_{12} \wedge e_{13}, e_{13} \wedge e_{15}, e_{14} \wedge e_{15}\right\}, & H_{8}=\left\{e_{12} \wedge e_{14}, e_{13} \wedge e_{15}, e_{14} \wedge e_{15}\right\}, \\
A=\left\{e_{12} \wedge e_{13}, e_{12} \wedge e_{14}, e_{13} \wedge e_{14}\right\}, & B=\left\{e_{12} \wedge e_{13}, e_{12} \wedge e_{14}, e_{12} \wedge e_{15}\right\} .
\end{array}
$$

Second, we introduce $T_{i} \subset \bigwedge^{i}(V)$ and $T_{i}(H) \subset \bigwedge^{i}(V)$ with $3 \leq i \leq 8$ by setting

$$
\begin{aligned}
T_{i} & =\left\{e_{\sigma} \in \bigwedge(V): h_{i-2} \wedge e_{12} \wedge e_{13}<_{\text {lex }} e_{\sigma}\right\} \\
T_{i}(H) & =\left\{h_{i-2} \wedge e_{\sigma}: e_{\sigma} \in H\right\} \quad \text { where } H \in\left\{H_{i}, A, B\right\} .
\end{aligned}
$$

Let $I=\bigoplus_{d=3}^{15} I_{d} \subset E$ denote the ideal of $E$ generated by the monomials belonging to $\bigcup_{i=3}^{8}\left(T_{i} \cup T_{i}\left(H_{i}\right)\right)$ together with all monomials of degree 9 and $\Delta$ the simplicial complex on $\{1, \ldots, 15\}$ with $I=J_{\Delta}$.

Lemma 4.1. (a) For $3 \leq d \leq 8$ the subspace $I_{d}$ is spanned by $T_{d} \cup T_{d}\left(H_{d}\right)$.

(b) Let $3 \leq d \leq 8$ and $e_{\sigma} \in I_{d}$ with $e_{\sigma} \notin T_{d}\left(H_{d}\right)$. Then $S_{i j}^{0}\left(e_{\sigma}\right)=e_{\sigma}$.

(c) Unless $12 \leq i<j \leq 15$ one has $S_{i j}^{0}\left(e_{\sigma}\right)=e_{\sigma}$ for all $e_{\sigma} \in \bigcup_{d=3}^{8} T_{d}\left(H_{d}\right)$.

Proof. (a) Let $3 \leq d<8$. We claim $e_{j}\left(T_{d} \cup T_{d}\left(H_{d}\right)\right) \subset T_{d+1}$ for all $j$. In fact, $h_{d-1} \wedge e_{12} \wedge e_{13}<_{\text {lex }} e_{j} \wedge h_{d-2} \wedge e_{p} \wedge e_{q}$ unless $e_{j} \wedge h_{d-2} \wedge e_{p} \wedge e_{q} \neq 0$.

(b) Let $e_{\sigma} \in I_{d}$ with $e_{\sigma} \notin T_{d}\left(H_{d}\right)$. Let $j \in \sigma$ and $i \notin \sigma$. Since $h_{d-2} \wedge e_{12} \wedge e_{13}<_{\text {lex }}$ $e_{\sigma}$, one has $h_{d-2} \wedge e_{12} \wedge e_{13}<_{\text {lex }} e_{(\sigma \backslash\{j\}) \cup\{i\}}$. Thus $e_{(\sigma \backslash\{j\}) \cup\{i\}} \in T_{d}$. Hence $S_{i j}^{0}\left(e_{\sigma}\right)=e_{\sigma}$.

(c) Let $i<12$. Let $e_{\tau}=h_{d-2} \wedge e_{\sigma} \in T_{d}\left(H_{d}\right)$. Let $j \in \tau$ and $i \notin \tau$. Then $h_{d-2} \wedge e_{12} \wedge e_{13}<_{\text {lex }} e_{(\tau \backslash\{j\}) \cup\{i\}}$. Thus $e_{(\tau \backslash\{j\}) \cup\{i\}} \in T_{d}$. Hence $S_{i j}^{0}\left(e_{\sigma}\right)=e_{\sigma}$.

Given a sequence $\mathbf{Q}=\left(Q_{3}, \ldots, Q_{8}\right)$ with each $Q_{i} \in\{A, B\}$ we write $I^{\mathbf{Q}}$ for the ideal of $E$ generated by the monomials belonging to $\bigcup_{i=3}^{8}\left(T_{i} \cup T_{i}\left(Q_{i}\right)\right)$ together with all monomials of degree 9 . Let $\mathcal{W}_{\text {shift }}(\Delta)$ denote the set of combinatorial shifted complexes of $\Delta$.

Lemma 4.2. (a) Let $\Delta^{c} \in \mathcal{W}_{\text {shift }}(\Delta)$. Then $J_{\Delta^{c}}$ is of the form $I^{\mathbf{Q}}$.

(b) None of $\Delta^{c} \in \mathcal{W}_{\text {shift }}(\Delta)$ satisfies $J_{\Delta^{c}}=I^{(A, \ldots, A)}$.

(c) None of $\Delta^{c} \in \mathcal{W}_{\text {shift }}(\Delta)$ satisfies $J_{\Delta^{c}}=I^{(B, \ldots, B)}$.

(d) For each $i$ and for each $j$ with $i<j$ there is $\Delta^{c}(i, j ; A) \in \mathcal{W}_{\text {shift }}(\Delta)$ with $J_{\Delta^{c}(i, j ; A)}=I^{\mathbf{Q}}$, where $Q_{i}=Q_{j}=A$.

(e) For each $i$ and for each $j$ with $i<j$ there is $\Delta^{c}(i, j ; B) \in \mathcal{W}_{\text {shift }}(\Delta)$ with $J_{\Delta^{c}(i, j ; B)}=I^{\mathbf{Q}}$, where $Q_{i}=Q_{j}=B$.

Proof. After repeated applications of the operations $S_{i_{k} j_{k}}^{0}$, where $12 \leq i_{k}<j_{k} \leq 15$ and where $k=1,2, \ldots$, each subset $T_{d}\left(H_{d}\right)$ will shift to either $T_{d}(A)$ or $T_{d}(B)$. 
Moreover, $S_{i j}^{0}\left(T_{d}(A)\right)=T_{d}(A)$ and $S_{i j}^{0}\left(T_{d}(B)\right)=T_{d}(B)$ for all $1 \leq i<j \leq 15$. Our claim (a) follows from this observation together with Lemma 4.1 .

A routine computation yields the classification of the sequences $\mathbf{Q}=\left(Q_{3}, \ldots, Q_{8}\right)$ for which there is $\Delta^{c} \in \mathcal{W}_{\text {shift }}(\Delta)$ with $J_{\Delta^{c}}=I^{\mathrm{Q}}$. The classification table is

$$
\begin{gathered}
(A, A, A, A, A, B),(A, A, A, A, B, A), \ldots,(B, A, A, A, A, A), \\
(B, B, B, B, B, A),(B, B, B, B, A, B), \ldots,(A, B, B, B, B, B)
\end{gathered}
$$

together with

$$
\begin{array}{ll}
(A, A, A, B, B, B), & (B, A, B, A, B, A), \\
(B, B, A, B, A, A), & (A, B, B, A, A, B) .
\end{array}
$$

Our claims (b), (c), (d) and (e) now follow immediately.

Theorem 4.3. (a) None of $\Delta_{\sharp}^{c} \in \mathcal{W}_{\text {shift }}(\Delta)$ satisfies $\beta_{i i+j}\left(J_{\Delta^{c}}\right) \leq \beta_{i i+j}\left(J_{\Delta_{\sharp}^{c}}\right)$ for all $\Delta^{c} \in \mathcal{W}_{\text {shift }}(\Delta)$ and for all $i$ and $j$.

(b) None of $\Delta_{b}^{c} \in \mathcal{W}_{\text {shift }}(\Delta)$ satisfies $\beta_{i i+j}\left(J_{\Delta_{b}^{c}}\right) \leq \beta_{i i+j}\left(J_{\Delta^{c}}\right)$ for all $\Delta^{c} \in$ $\mathcal{W}_{\text {shift }}(\Delta)$ and for all $i$ and $j$.

Proof. Let $\Delta_{\sharp}^{c} \in \mathcal{W}_{\text {shift }}(\Delta)$ with $J_{\Delta_{\sharp}^{c}}=I^{\mathrm{Q}}$. By Lemma 4.2 (c) there is $3 \leq j \leq 8$ with $Q_{j}=A$ and $Q_{j^{\prime}}=B$ for all $3 \leq j^{\prime}<j$. Lemma 4.2 (e) guarantees the existence of $\Delta^{c}(j-1, j ; B) \in \mathcal{W}_{\text {shift }}(\Delta)$ with $J_{\Delta^{c}(j-1, j ; B)}=I^{\mathbf{Q}^{\prime}}$, where $\mathbf{Q}^{\prime}=\left(Q_{3}^{\prime}, \ldots, Q_{8}^{\prime}\right)$ with $Q_{j-1}^{\prime}=Q_{j}^{\prime}=B$. Then for $i \neq 14$ one has

$$
m_{\leq i}\left(J_{\Delta^{c}(j-1, j ; B)}, j-1\right)=m_{\leq i}\left(J_{\Delta_{\sharp}^{c}}, j-1\right)
$$

and

$$
m_{\leq i}\left(J_{\Delta^{c}(j-1, j ; B)}, j\right)=m_{\leq i}\left(J_{\Delta_{\sharp}^{c}}, j\right) .
$$

On the other hand, $m_{\leq 14}\left(J_{\Delta^{c}(j-1, j ; B)}, j-1\right)=m_{\leq 14}\left(J_{\Delta_{\sharp}^{c}}, j-1\right)$ and $m_{\leq 14}\left(J_{\Delta^{c}(j-1, j ; B)}, j\right)<m_{\leq 14}\left(J_{\Delta_{\sharp}^{c}}, j\right)$. Now, Lemma 2.8 says that $\beta_{i i+j}\left(J_{\Delta_{\sharp}^{c}}\right)<$ $\beta_{i i+j}\left(J_{\Delta^{c}(j-1, j ; B)}\right)$ for all $i$. Thus $\Delta_{\sharp}^{c} \in \mathcal{W}_{\text {shift }}(\Delta)$, such that $\beta_{i i+j}\left(J_{\Delta^{c}}\right) \leq \beta_{i i+j}\left(J_{\Delta_{\sharp}^{c}}\right)$ for all $\Delta^{c} \in \mathcal{W}_{\text {shift }}(\Delta)$ and for all $i$ and $j$, does not exist. This completes the proof of (a). A similar technique can be used to prove (b).

Corollary 4.4. None of $\Delta^{c} \in \mathcal{W}_{\text {shift }}(\Delta)$ satisfies $\Delta^{e}=\Delta^{c}$.

Proof. Let $\Delta_{b}^{c} \in \mathcal{W}_{\text {shift }}(\Delta)$ satisfy $\Delta^{e}=\Delta_{b}^{c}$. Since $\beta_{i i+j}\left(J_{\Delta^{e}}\right) \leq \beta_{i i+j}\left(J_{\Delta^{c}}\right)$ for all $i$ and $j$, it follows that $\beta_{i i+j}\left(J_{\Delta_{b}^{c}}\right) \leq \beta_{i i+j}\left(J_{\Delta^{c}}\right)$ for all $\Delta^{c} \in \mathcal{W}_{\text {shift }}(\Delta)$ and for all $i$ and $j$. This fact contradicts Theorem 4.3 (b). Thus none of $\Delta^{c} \in \mathcal{W}_{\text {shift }}(\Delta)$ satisfies $\Delta^{e}=\Delta^{c}$, as desired.

\section{REFERENCES}

[1] A. Aramova, J. Herzog and T. Hibi, Squarefree lexsegment ideals, Math. Z. 228 (1998), $353-$ 378. MR1630500 (99h:13013)

[2] A. Aramova, J. Herzog and T. Hibi, Gotzmann theorems for exterior algebras and combinatorics, J. of Algebra 191 (1997), 174 - 211. MR1444495 (98c:13025)

[3] A. Aramova, J. Herzog and T. Hibi, Shifting operations and graded Betti numbers, J. Algebraic Combin. 12 (2000), 202 - 222. MR.1803232 (2001k:13022)

[4] W. Bruns and J. Herzog, "Cohen-Macaulay rings," Revised Edition, Cambridge University Press, 1996. MR1251956 (95h:13020)

[5] P. Erdös, C. Ko and R. Rado, Intersection theorems for systems of finite sets, Quart. J. Math. Oxford Ser. (2) 12 (1960), 313 - 320. MR0140419 (25:3839) 
[6] M. Green, Generic initial ideals, in "Six Lectures on Commutative Algebra" (J. Elias, J. M. Giral, R. M. Miró-Roig and S. Zarzuela, Eds.) Birkhäuser, 1998, pp. 119 - 186. MR1648665 (99m:13040)

[7] J. Herzog, Generic initial ideals and graded Betti numbers, in "Computational Commutative Algebra and Combinatorics" (T. Hibi, Ed.), Advanced Studies in Pure Math., Volume 33, 2002, pp. 75 - 120. MR.1890097 (2003b:13021)

[8] G. Kalai, Algebraic shifting, in "Computational Commutative Algebra and Combinatorics" (T. Hibi, Ed.), Advanced Studies in Pure Math., Volume 33, 2002, pp. 121 - 163. MR1890098 (2003e:52024)

Department of Pure and Applied Mathematics, Graduate School of Information Science and Technology, Osaka University, Toyonaka, Osaka 560-0043, Japan

E-mail address: s-murai@ist.osaka-u.ac.jp

Department of Pure and Applied Mathematics, Graduate School of Information Science and Technology, Osaka University, Toyonaka, Osaka 560-0043, Japan

E-mail address: hibi@math.sci.osaka-u.ac.jp 